\title{
Suspensão Diária da Sedação: Uma Panacéia?
}

\author{
Daily Interruption of Sedatives: A Panacea?
}

Rachel Duarte Moritz

É indiscutível que tanto a sedação excessiva quanto a agitação dos pacientes internados em unidades de terapia intensiva (UTI) são fatores indesejáveis. Também não existe dúvida de que a supersedação pode resultar em maior tempo de ventilação mecânica (VM) e de internação nas UTI, fatos que podem interferir negativamente na evolução dos pacientes críticos.

Após o estudo de Kress e col. ${ }^{1}$, tem tido aceitação crescente a importância da suspensão diária da sedação para a avaliação da possibilidade do desmame da VM. Girard e col${ }^{2}$. avaliaram 336 pacientes submetidos à VM. Os autores arrolaram os pacientes intubados havia 12 horas, com pressão positiva de $8 \mathrm{cmH}_{2} \mathrm{O}$, fração inspirada de oxigênio de 0,5 , saturação de oxigênio maior que $88 \%$ e necessitando de doses moderadas de vasopressores. Os pacientes foram separados em 2 grupos: controle (GC) e estudo (GE). Os pacientes do GC permaneciam sendo medicados com sedativos e, se estivessem ventilando espontaneamente, eram submetidos ao desmame em tubo- $\mathrm{T}$ ou pressão de suporte $\leq 7 \mathrm{cmH}_{2} \mathrm{O}$ ou pressão controlada $\leq 5 \mathrm{cmH}_{2} \mathrm{O}$ Os pacientes eram avaliados por 120 minutos. Daqueles selecionados para o GE foram retirados os fármacos sedativos e após 4 horas, se os pacientes acordassem ao estímulo oral e apresentassem drive respiratório era iniciado o desmame de forma semeIhante ao GC. Os autores concluíram que os pacientes aos quais foi suspensa a sedação, embora tenham apresentado maior incidência de auto-extubação, permaneceram menos tempo sem VM, menos tempo internados na UTI e no hospital e, após um ano, esses pacientes apresentaram menor probabilidade de morte.

Brochard $^{3}$ comenta o referido artigo, aponta algumas falhas e conclui que são necessárias novas informa-

Coordenadora da Residência de Medicina Intensiva do Hospital Universitário da Universidade Federal de Santa Catarina; Mestre em Ciências Médicas; Doutora em Engenharia de Produção

Endereço para correspondência:

Rua João Paulo 1929 - Bairro João Paulo

88030-300 Florianópolis, SC

E-mail-rachel@hu.ufsc.br

(C)Associação de Medicina Intensiva Brasileira, 2008 ções que comprovem a eficácia da suspensão diária de sedativos. O autor afirma que o uso indiscriminado dessa técnica pode ser danoso para os pacientes que necessitam de sedação.

Diante do exposto, considero importante tecer alguns comentários. Acredito que o grande benefício dos estudos que defendem a suspensão diária da sedação é o de que, nos permitem a constatação de que atualmente existe a tendência dos intensivistas sedarem demais os seus pacientes e, conseqüentemente retardarem o seu desmame.

Essa constatação me permite a defesa de que, mais importante do que a suspensão indiscriminada e diária da sedação, é o médico intensivista ao lado do paciente. Sem sombra de dúvida a elaboração de protocolos é importante para direcionar uma terapêutica. Entretanto, jamais poderá substituir o médico ao lado do paciente, avaliando-o de forma ampla e questionando diariamente: Esse paciente pode ser desmamado da VM? Está adequadamente analgesiado? Necessita ser sedado? Qual será a sedoanalgesia ideal para ele? Como está o seu quadro clínico. Como iremos otimizar o seu tratamento? Como nós intensivistas sabemos, o quadro clínico do paciente crítico é bastante complexo de ser definido. Como um corolário, as opções terapêuticas devem ser tomadas após raciocínio clínico adequado e não somente baseadas em rotinas anexadas no pé da cama dos pacientes.

Concluo esse comentário, fazendo minhas as palavras de Fletcher4 "On the other hand, evidence-based Medicine is meant to complement, not replace, clinical judgment failure".

\section{REFERÊNCIAS}

01. Kress JP, Pohlman AS, O'Connor MF et al - Daily interruption of sedative infusions in critically ill patients undergoing mechanical ventilation. $\mathrm{N}$ Engl J Med, 2000;342:1471-1477.

02. Girard TD, Kress JP, Fuchs BD et al - Efficacy and safety of a paired sedation and ventilator weaning protocol for mechanically ventilated patients in intensive care (Awakening and Breathing controlled trial): a randomized controlled trial. Lancet, 2008;371:126-134.

03. Brochard L - Sedation in the intensive-care unit: good and bad? Lancet, 2008;371:95-97.

04. Fletcher RH - Up-To-Date; this topic was last changed on August 23, 2007 (acessado em janeiro de 2008). 